\title{
Decision Making Tool for the Selection of Urban Freight Transport Project
}

\author{
Nicolas Malhéné and Dominique Breuil \\ EIGSI - Ecole d'Ingénieurs en Génie des Systèmes Industriels, \\ 26, rue des Vaux de Foletier La Rochelle Cedex 117041 \\ \{nicolas.malhene, dominique.breuil\}@eigsi.fr
}

\begin{abstract}
The control of transport is a very current preoccupation in a context of sustainable development. Nuisances associated to Urban Freight Transport (UFT) force politicians to propose measures relevant to sustainable development of the city. Nevertheless they should keep in mind the efficiency of UFT which renders the implementation of new projects quite difficult. We propose to transpose systemic approach to UFT problematic and to develop a global approach for the management of the evolution of City Logistics. Our first propositions in the domain conduce to the development of a tool allowing politicians to determine UFT solution coherent with their objectives.
\end{abstract}

Keywords: Urban Freight Transport, Evolution, innovative solutions for UFT.

\section{Introduction}

COST 321 Actions [1] show that freight transport contributes about $20 \%$ in the total traffic within urban areas but represents $40 \%$ of the pollution. Recent and convergent surveys (ex "Sustainable Urban Transport Plans" in 2007, French national surveys in 2004 or Bestufs indicators) gave UFT's quantified situation in the city flows and its evolutions in next coming years; the growing importance of these flows will be more and more worrying. The importance of UFT has also been highlighted by the cost distribution within the freight transport chain. The weight of these costs is further increased by the reduction of stocks, the smaller size of consignments and the increase in their number [2]. UFT challenges can be classified in four categories [3]:

- Functional ones address the city as a whole and more particularly the integration of goods flows in global sustainable transport plans and the sharing transport facilities with passengers flows;

- Economics, since UFT efficiency directly impacts the performances of supply chains and influences indirectly the commercial or crafts activities of a city;

- Urban planning ones because the frequentation and the occupation of space are indeed closely related to his accessibility both for the people and the goods;

- Societal and environmental ones have direct effects on the quality of life.

Considering resulting complexity, it is quite difficult for politicians to optimize UFT. Furthermore, even if they succeed in implementing a so called optimized UFT 
system, the fast evolution of the economic environment makes this system quickly obsolete. The evolution of UFT system must be controlled together with the evolution of its environment. The first part of the paper proposes a reference framework to study UFT in medium size cities and to formalize the associated problematic. The second part shows how innovative works relevant to the production systems domain may be transferred towards UFT systems in order to conceptualize the management of their evolution. The last part of the paper presents the prototype of a software tool to support such approach.

\section{Reference Framework for the Study of Urban Freight Transport}

\subsection{Concept of System}

The framework should be global in order to include all stakeholders, resources, constraints associated to UFT and to underline relationships between previous elements. Widely exploited in production systems domain, systemic approach fits our criteria. It proposes a conceptual framework, a set of knowledge and tools to understand phenomena in a global way. A system is defined as a group of elementary processors of various natures connected and interconnected, in order to satisfy one or several defined objectives or finalities.

By definition, the environment of the system contains all that the modeler does not wish to integrate into the system. In particular, the environment contains the entity which wishes to consume for its own activity one or several Objects that it can not produce itself. The identification of such a relation brings to the foreground a system.

\subsection{Characterization of the UFT System}

To transpose systematic paradigm into the urban transport problematic, we could define the environment of our system as being composed of all the entities wishing to be moved by a $A$ point towards a $B$ point at time $t$ given. In our proposition, $A$ or/and $B$ correspond(s) to location(s) in the city when entity is a specific good. Our environment wishes to consume an Object which we shall define as the service of transport necessary for this movement. Thus UFT Transport system is depicted through fig. 1:

Systematic paradigm [4] stresses the need for a system to be able to evolve in order to adapt itself permanently to the modifications of its environment. The system's variety is function of the potential number of relations between system's elementary processors and depends directly on the number of processors of the system. By the way, variety represents the capability of the system to evolve. One closed system can not import or exchange processors: its variety growths till a maximum $V_{\max }=2^{\mathrm{N}^{2}}$ where $\mathrm{N}$ is the number of processors of the system. This value determines the end of the evolution process. This is not the case for an open system: the variety of the system continues to grow while the system is able to import new processors. On an etymologic point of view, the system should stay a coherent group in order to fulfill the finality. The importation and the integration of new processors have to be controlled then the evolution of the system can be managed. 


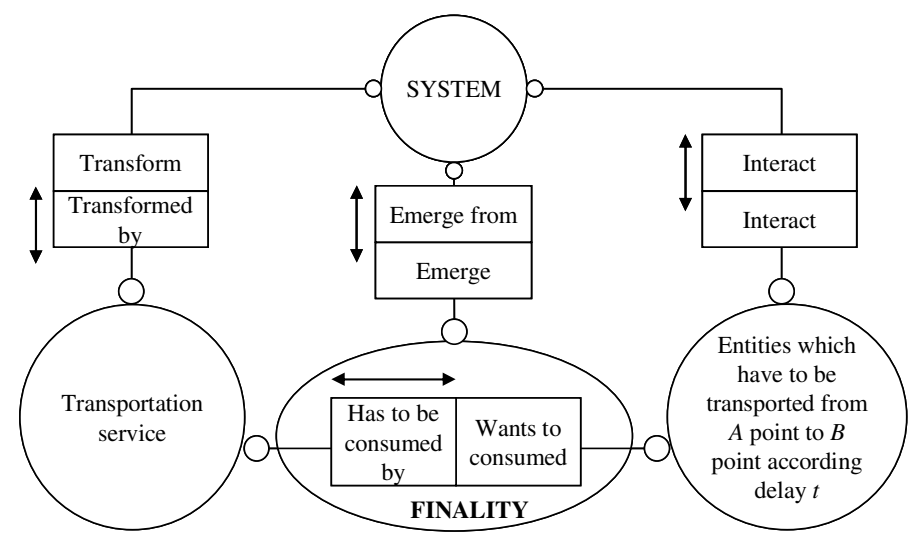

Fig. 1. Emergence of the system dedicated to transport of entities

\section{Management of the Evolution of UFT System}

\subsection{Conceptual Approach of the Evolution Trajectory}

Our work is mainly based on techniques developed by the GRAI Laboratory on the coherence analysis and on the performance evaluation in manufacturing systems [5]. This research conduces to characterize the evolution of the system as a continuous process based on a combination of "steps". Each step represents the evolution of the status of the system [6].

SHOULD-BE corresponds to the idealist vision of the UFT system at time $t$. It is expressed through the vector of operational performances expected by stakeholders. Associated to a strategic horizon this state will not be reached; during this lead time system environment will be modified requiring definition of different vector of performances. AS-IS corresponds to representation of the UFT system at time $t$. It gives a model of actual UFT system as well as the actual vector of operational performances. Successive STEP-n draw the evolution trajectory of the UFT system. The

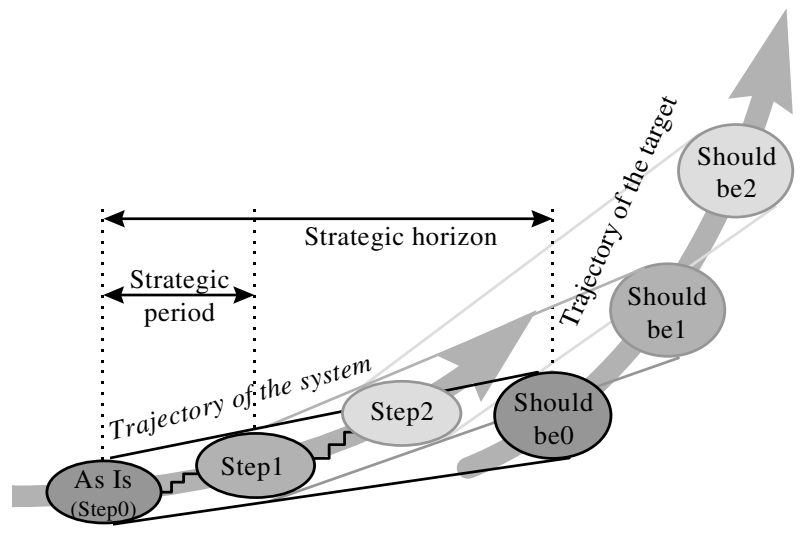

Fig. 2. Evolution process 
interval of time existing between two successive steps is the strategic period. It corresponds to the lead time at the end of which the UFT system must reconsider its trajectory (adaptation of its objectives with the evolution of the environment).

The evolution process depicted through these different conceptual states underlines three levels of management.

- The Strategic Definition level is based on the gap between the AS-IS and the SHOULD-BE in term of performances. Strategic decisions design the trajectory defining different STEP-n associated to coherent levels of performance which lead to the SHOULD-BE;

- The Actions Planning level operates in a world of models. Once existing UFT system has been modeled (AS IS), the design phase consists in modifying this model arranging processors interconnections and introducing new processors. This phase aims at giving the future model (STEP-1) the potential that ensure the UFT system to match operational performances provided by upper level. Actions Planning decisions aim at linking on one side the gap of operational performance between existing and future system and on the other side design objectives;

- The Projects Management level has in charge the integration of new processors. This level corresponds to a classical multi-project management system and then all activities usable for such a management find their place within it and in particular activities referring to human resources involvement.

\subsection{Proposal for a Formalization of Performance-Based Management of Evolution}

The Strategic Definition level operates only in term of performances evolution. The UFT system can be modeled in a space of operational performances. CIVITAS program (cf www.civitas-initiative.eu) identified different performance criteria for such system: Operating costs, Air Quality, Service reliability, Congestion Levels, etc. This level draws the evolution of the system through a vector of performances. For instance, a UFT system is chosen to be evaluated through three performances: $\mathrm{P}_{1}, \mathrm{P}_{2}$ and $\mathrm{P}_{3}$. Then the system itself and its evolution will be modeled inside a threedimension space (Fig. 3).

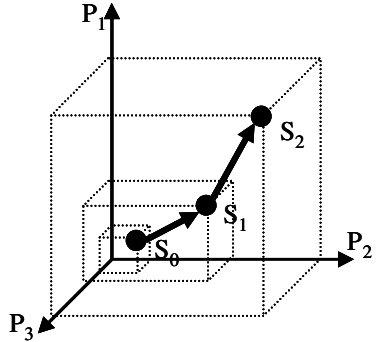

State trajectory in the space of performances

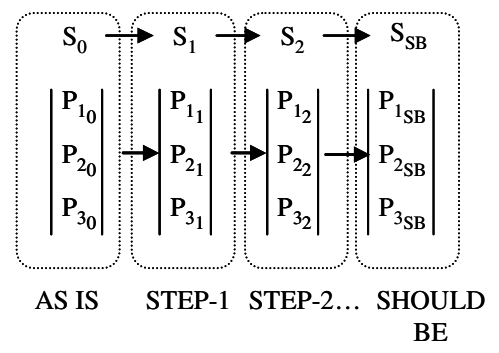

Evolution of the system as an evolution of its performances vector

Fig. 3. Modeling the system inside a space of performances 
During the evolution process, the repository of performance does not change: the criteria are kept and only their value is modified. If it was necessary to modify it repository, it would mean that the objectives of evolution changed and that a new process must be engaged.

\subsubsection{Dynamics and Characterization of Evolution}

Each evolution state is associated to a vector of performances. Two following states are linked by a step of evolution called "transition". A transition is characterized by a cost value (regarding one or several criteria like money, environment or social impacts ...) and a time to be fulfilled. cost value and time are strongly linked.

\subsubsection{Accessibility}

An evolution state is accessible if the associated vector of performances can exist, i.e. if the variables of the vector respect internal and external constraints. Two main kinds of situations explain this notion:

- The first one corresponds to a level of performance which cannot be reached by one or several variables of the vector (for instance, the $\mathrm{CO}_{2}$ emission variable is likely to be limited by $0 \mathrm{mg}$ regarding the vehicle life cycle);

- The second situation corresponds to the case where one variable of the vector limits other ones (mutual exclusion). The case in which two performances are linked by a trade-off illustrates this situation. As an example, experiments demonstrate that Operating costs and Service reliability limit each other.

\subsubsection{Pertinence}

A state is pertinent if each performances vector variable participates effectively to the global performance expected by the enterprise. Effective participation of a variable means that the reduction of its value induces global performance reduction.

\section{Decision Aided Tool to Support UFT Evolution Process}

Our previous theoretical reflections represent the first step for the development of a global approach. Nevertheless to interest politicians and other stake holders to these concepts, it is important to present operational results. For this reason we have initiated the development of a software tool which can support our approach. We had first to identify what are the processors or relevant innovative solutions allowing UFT system evolution.

\subsection{Relevant Processors or Innovative Solutions in UFT}

UFT's solutions have been studied and experimented for quite some time now. Different categories of innovative solutions have been identified and many of them have been recorded in the European BESTUFS program [7]; several demonstration measures have been conduced in the frame of CIVITAS initiative European program. In France several studies and some solutions have been supported by the PREDIT [8]. Short or medium term evolutions can already be foreseen; some of them linked to the 
deployment of telematics, others due to development of technologies or of awareness of the stakeholders:

\subsubsection{To Develop Infrastructures}

This category of solutions reefers to all types of infrastructures. Various actions are engaged from anticipation in land use planning to the adaptation of dedicated lanes. Evolutions will concern mainly access controlled zones and delivery bays. For both of them, new concepts such as status variation during the day (i.e. adapting the finality of the road space during the day) or new technologies (ex. remote control of delivery bays occupation) will bring flexibility in the utilization of the space.

\subsubsection{To Facilitate City's Access}

Solutions concern all information devices which can be thought of in order to make easier the drivers' job to deliver products in the city. Then signing equipments as well as software participate to these improvements which are linked directly to the progresses of Information \& Telecommunication Technologies. In the very next future, specific routing messages will be transmitted to drivers to modify their routes according to traffic situation or global goods distribution will be organize through common information platform shared by haulers. This will also concern the future self routing determination that RFID equipped products will have in the future.

\subsubsection{To Implement Proximity Storage Facilities}

This topic concerns all types of storage from freight villages' warehouses to logistic boxes, going through Urban Freight Consolidation Centre platforms. Many experimentation cases already showed the various ways of organizing distribution from these facilities. The main evolutions will concern both the management and the global organization of these facilities in the cities, in order to optimize the efficiency of distribution activities and make them really profitable. Then the different types of proximity storages could be combined according to the surroundings activities, on permanent or temporary basis (building construction, specific events...).

\subsubsection{To Use Adequate Vehicles}

Important developments are yet to be realized in that domain; Apart from existing clean vehicles which are more or less fit for city logistics, new ones are already designed and should be used in the next coming years. Other concepts will be implemented, probably in a combined way in order to adapt the vehicles to the transported goods, final destination and cost effectiveness. Of course these developments are related to the organization of storage facilities.

\subsubsection{To Control}

To control means both to set up adapted, homogeneous and coherent regulations and the verification of their compliance by concerned stakeholders. The main evolutions will concern more the way the results are analyzed and used for evaluation purpose of other actions than on the regulations themselves for which almost all possible experimentations have been realized. 


\subsubsection{To Set Up Partnerships}

This point is the key factor which is necessarily part of other improvements. This cannot be set alone, for whatever the negotiations between stakeholders deal with, there will be concrete modifications in the goods circulation. Several types of partnerships' building strategies have been experimented (ex FQP, Freight Quality Partnerships in England) involving main transport companies delivering goods in cities. The evolution will be on the ways to involve all stakeholders in the partnerships or the incentive given to increase their motivation.

\subsection{Decision Tool for the Importation of UFT Processor}

Our objective is to develop a tool which can support the evolution of UFT system. The first version does not aim at integrating all our theoretical proposals. It focuses on two consecutive steps and the associated transition. Respecting principles of accessibility and pertinence, the resulting operational tool aims at directing politicians to several projects, so that they become aware of the largest number of potential solutions. The tool is based on a data base and a qualitative matrix. The data base includes the results of CIVITAS initiative European programme in particular:

- Characteristics of different middle size cities involved in the program,

- Characteristics of several demonstration measures launched by those cities.

The set of city's characteristics corresponds to a basic model of the city. It also corresponds to a vector of performances which guides the evolution of UFT system. Based on these elements, the use of the tool is quite easy. First, the decision maker has to input the characteristics of his city. These characteristics are compared to average CIVITAS data. A score results from this comparison and allows going through the qualitative matrix which is already fulfilled.

This matrix is the kernel of the tool. To highlight the most sensible solution to be set up in a city, we opted for a homemade binary notation. This system of notation allows to increase the note associated to a solution if its contribution is considered as positive with regarding a specific city characteristic. If its contribution is negative or neutral the note is not influenced. By the way the biggest note corresponds to the most pertinent solution in this sense that it positively influences a majority of the city characteristics.

So, the tool informs the decision maker on the opportunity to choose a UFT action among others through a hierarchical presentation. Finally, the decision making tool presents the results of the implementation of similar action in a similar context. It also gives information related to characteristics, advantage and inconvenient of such actions as well as the cost aspects considering the fact that such factor is quite important for this kind of decision.

\section{Conclusion}

The tool has been validated by politicians of the Urban Community of La Rochelle. It is a first version of a global set of tools which will integrate all the conceptual approach and relevant theoretical proposals. The overall approach itself and in particular 
the formalization of the evolution process will be widely developed. Recent work related to management of the evolution process of industrial enterprise gives some perspectives. [9] conceptualizes change projects to evaluate associated cost, duration and impacts. The generic framework developed in this work allows evaluating the interest of change projects in order to build a relevant trajectory. This approach could be adapted to our problematic. This will be the next step before considering the global optimisation of urban mobility. In this perspective, an integrated approach will deal with both UFT and Transport of Passengers opening research field toward interoperability and system of systems.

\section{References}

1. COST 321 Action, European Commission Directorate General Transport (1998) COST 321 Urban Goods Transport, final report, Brussels (European Commission) (1998)

2. Study material, Inner Urban Freight Transport and City Logistics (2003), http: / /www. euportal. net

3. Boudouin, D., Morel, C.: Logistique Urbaine - l'optimisation de la circulation des biens et services en ville. In: Programme national "Marchandises en ville", Documentation Française, p. 15 (2002)

4. Le Moigne, J.L.: La théorie du système général. Théorie de la modélisation, Presses Universitaires de France, Paris (1977)

5. Ducq, Y.: Contribution à une méthodologie d'analyse de la cohérence des systèmes de production dans le cadre du modèle GRAI, Thèse de doctorat, Université Bordeaux 1, Février (1999)

6. Malhéné, N.: Gestion du processus d'évolution des systèmes industriels - Conduite et méthode, Thèse de doctorat, Université Bordeaux 1 (Janvier 2000)

7. BESTUFS, Good Practice Guide (September 2007), http : / /www . bestufs . net/

8. PREDIT, Publications of Operational Group no. 5 (2008) Logistics and Freight Transport, http: / / www . predit.prd. fr/predit 3 / goDirect. fo? cmd=go\&inCde=5

9. Ben Zaïda, Y.: Contribution à la conduite du changement du système entreprise, Thèse de doctorat, Université Montpellier 2 (Novembre 2008) 